\title{
Modelling Dynamics of Genetic Networks as a Multiscale Process
}

\author{
Xilin Wei, Roderick V.N. Melnik, and Gabriel Moreno-Hagelsieb \\ Mathematical Modelling and Computational Sciences, \\ Wilfrid Laurier University, \\ 75 University Ave W, Waterloo, Ontario, N2L 3C5, Canada
}

\begin{abstract}
A key phenomenon in the dynamics of genetic networks is the cell cycle. In the study of this phenomenon, an important task is to understand how many processes, acting on different temporal and spatial scales, interact in the cell.

In this paper we deal with the problem of modelling cell cycles. We start our analysis from the Novak-Tyson model and apply this deterministic model to simulate relative protein concentrations in several different living systems, including Schixosaccharomyces pombe to validate the results. Then we generalize the model to account for the nonlinear dynamics of a cell division cycle, and in particular for special events of cell cycles. We discuss the obtained results and their implications on designing engineered regulatory genetic networks and new biological technologies.
\end{abstract}

\section{Introduction}

Cells process information in complex ways. During the cell cycle, an eukaryotic cell duplicates all of its components and separates them into two daughter cells. This process is composed of four phases: G1 phase in which size of the cell is increased by producing RVA and synthesizing protein, S phase in which DNA are replicated, G2 phase in which the cell continues to produce new proteins and grows in size, and M (mitosis) phase in which DNA are separated and cell division takes place [1], 3]. From the outset, we are in a situation where we have to deal with different biological events with different spatial and temporal scales.

The problem of modelling dynamics of genetic networks, including those for cell cycles, has been actively addressed in the past decades [2. New improved models have been recently developed with increasing capability to predict competitively experimental results [3]. The Novak-Tyson model for a cell cycle in [3] contains over 40 parameters that are of the same units but vary from less than $10^{-2}$ to 35 . A stochastic generalization of that model was presented in 4 .

In the present work, we start our analysis from the Novak-Tyson model and apply this deterministic model to simulate relative protein concentrations in several different living systems. Then, we generalize the model to account for the nonlinear dynamics of a cell division cycle, and in particular for special 
events of cell cycles. We show that the effects of such fluctuations may have important implications on designing engineered regulatory genetic networks due to the sensitivity of the model to parametrization processes.

\section{Mathematical Models of Cell Cycles}

Based on the original Novak-Tyson model, in this section, we develop a new model that accounts for fluctuations of concentrations in response to the multiscale character of cellular activities.

\subsection{The Novak-Tyson Model}

With $x_{1}(t)=C d c 13_{T}(t), x_{2}(t)=\operatorname{preMPF}(t), x_{3}(t)=S t e 9(t), x_{4}(t)=S \operatorname{Si} 1_{T}(t)$, $x_{5}(t)=S l p 1(t), x_{6}(t)=I E P(t), x_{7}(t)=R u m 1_{T}(t), x_{8}(t)=S K(t)$ and $M P F(t)$ denoting the relative concentrations of the corresponding proteins, and $x_{9}(t)=M(t)$ the mass of the cell in the cell cycle, the equations and parameters in the Novak-Tyson model are given in Table 1 where the time $t$ for variables $x_{i}, i=1,2, \ldots, 9 ; M P F, T F$, Trimer and $\Sigma$ is dropped.

Table 1. The Novak-Tyson Model. All constants have units $\min ^{-1}$, except the $J$ 's and $K_{\text {diss }}$ which are dimensionless

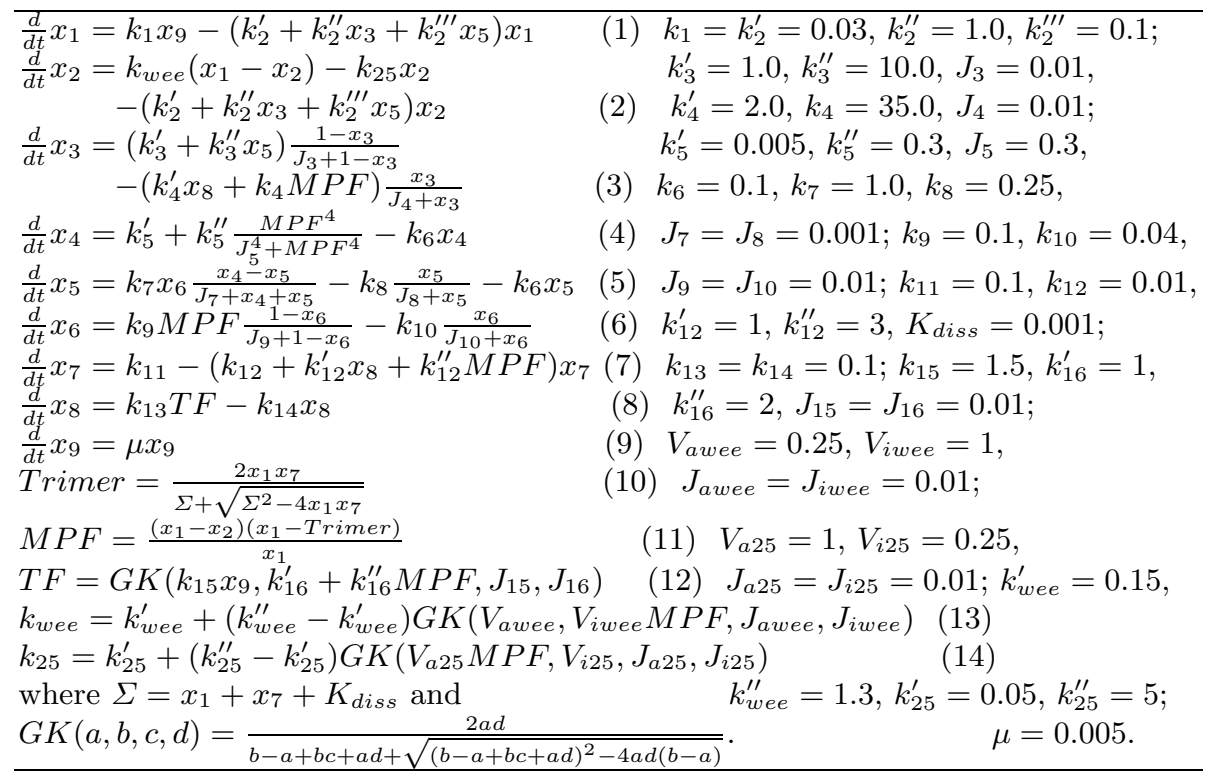

\subsection{The Generalized Model with Fluctuations}

Since a cell cycle involves nonlinear changes of the protein concentrations related to multiple spatial and temporal scales, the regulation of cellular activities 
contains a degree of uncertainty [3], 4]. Specifically, at the G1 phase, Ste9 and Rum 1 are activated while $S l p 1$ and $C d c 13_{T}$ are reducing rapidly. From the results of the deterministic model and experimental observations, the magnitudes of $S t e 9, C d c 13_{T}$ and $S l p 1$ are large enough to introduce fluctuations and the fluctuations of their derivatives are expected. $S K$ is also active at the latter part of the G1 phase. During the S phase which is shorter than G1 and G2 phases but much longer than $\mathrm{M}$ phase, the magnitudes of $C d c 13_{T}$ and preMPF are large enough to generate fluctuations of their changing rates. During the G2 phase, the magnitudes of $C d c 13_{T}$ and preMPF continue to increase. In the $\mathrm{M}$ phase, the magnitudes of $C d c 13_{T}$, pre $M P F$ and $\operatorname{slp} 1$ changes rapidly and are large enough to introduce fluctuations. IEP is also active in the $\mathrm{M}$ phase.

If the magnitude of the relative concentration of a protein $x_{i}(t)$ is beyond certain value (we use 0.3 for such a value in this paper), we need to modify the right hand sides (RHSs) of equations (1)-(9). Based on the experimental results (see Fig. 1) and taking into account that the period of the cell cycle is about $T=138.63$ minutes 3 , we suggest to multiply the RHSs of equations (1)-(9) by the functions $f_{1}(t), f_{2}(t), \ldots, f_{9}(t)$ respectively, where

$$
\begin{aligned}
& f_{j}(t)=\left\{\begin{array}{l}
1+r, k T \leq t \leq k T+\alpha_{j} \text { or } k T+\beta_{j} \leq t \leq(k+1) T ;, j=1,5 ; \\
1.0, \text { otherwise, }
\end{array}\right. \\
& f_{\ell}(t)=\left\{\begin{array}{l}
1+r, k T+\gamma_{\ell} \leq t \leq k T+\lambda_{\ell} ; \\
1.0, \text { otherwise }
\end{array}, \ell=2,3,6,8 ;\right. \\
& f_{4}(t)=f_{7}(t)=1.0 ; f_{9}(t)=1+r,
\end{aligned}
$$

$k$ is a nonnegative integer, $r$ is a control parameter that provides us with the amplitude of fluctuations, $\alpha_{1}=3, \beta_{1}=20, \alpha_{5}=15, \beta_{5}=T-5, \gamma_{2}=10$, $\lambda_{2}=T, \gamma_{3}=0, \lambda_{3}=20, \gamma_{6}=T-10, \lambda_{6}=T, \gamma_{8}=10$ and $\lambda_{8}=20$. Note that the choice of $f_{i}(t)$ for $i=1, \ldots, 9$ is not unique, but the above choice for $r=0$ is consistent with experimentally confirmed results of [3].

\section{Computational Experiments}

Both models, described in the previous section, have been implemented in MATLAB. We applied stiff solvers to deal efficiently with numerical difficulties caused by variability of model parameters. The initial conditions in all experiments are $x(0)=(0.45,0,1.0,0,2.1,0,0.05,0,1.0)$. In our first set of experiments, we use the deterministic Novak-Tyson model. The results with this model are presented in Fig. 1. Here and in all figures that follow we present two cycles. We observe that the relative concentrations of proteins are qualitatively the same as those obtained in [3], given differences of initial conditions. Replacing $k_{\text {wee }}^{\prime \prime}$ (parameters $k_{w e e}^{\prime \prime}$ and $k_{25}^{\prime \prime}$ are responsible for rate of tyr-phosphorylation and dephosphorylation) by 0.3 in the above model as suggested in [3], we get a model for the cell cycle of $W e e 1^{-}$mutants. The results obtained in this case are presented in Fig. 2. We can see that the relative concentrations of $C d c 13_{T}, M P F$ and preMPF 

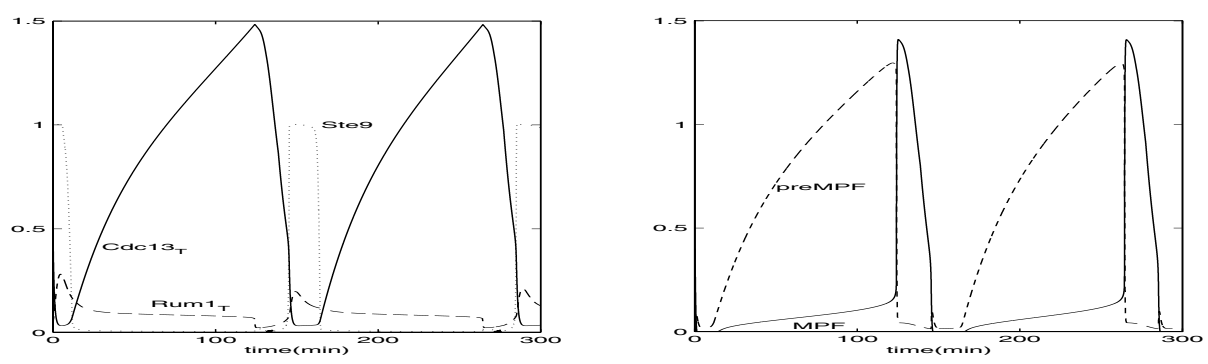

Fig. 1. Numerical simulation of the model in Section 2.1
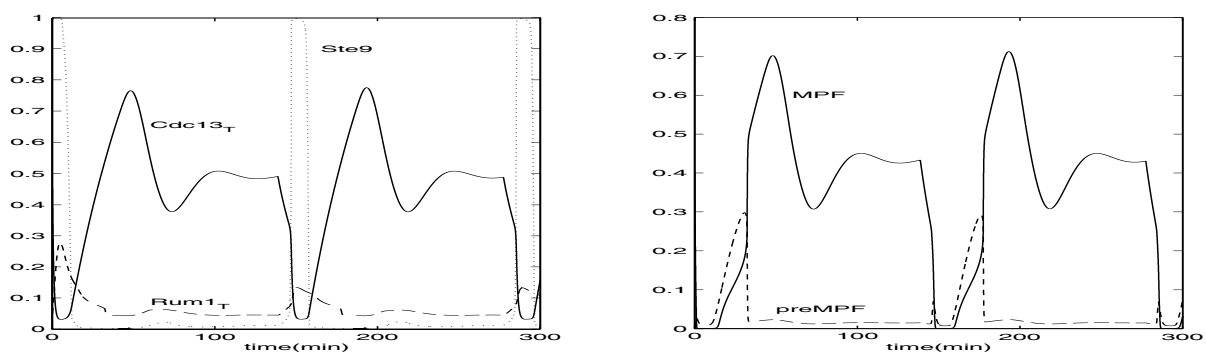

Fig. 2. Numerical simulation of the model with $k_{w e e}^{\prime \prime}=0.3$
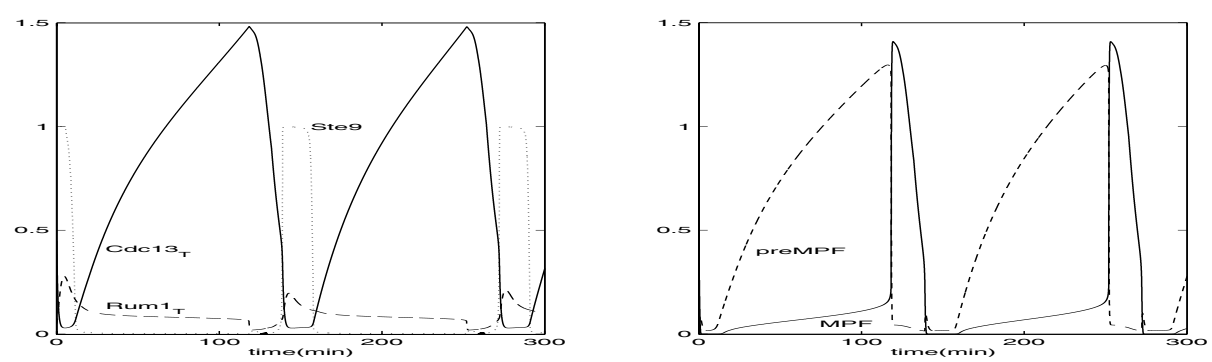

Fig. 3. Numerical simulation of the generalized model with $r=0.05$

in Fig. 2 are quite different from those in Fig. 1. We have also analyzed the situations with $k_{w e e}^{\prime \prime}=0.3$ and $k_{25}^{\prime \prime}=0.02$, as well as with $k_{25}^{\prime \prime}=0.02$, keeping $k_{w e e}^{\prime \prime}$ the same as in our first model. In both cases, noticeable changes in relative $M P F$ were observed.

In our second set of experiments, we use the generalized model given in Section 2.2. Setting sequentially $r=0.001, r=0.005, r=0.01$ and $r=0.05$ in (15)-(17), we obtained cell cycles with reduced cycle times. The results for two cycles for $r=0.05$ are shown in Fig. 3. They demonstrate that it is possible to regulate the cell cycle by adjusting the perturbation control parameter $r$. 


\section{Conclusions}

In this paper, we proposed a new model of cell cycle processes. The model takes into account special events during the cell cycle. The developed methodology can also be used to guide investigations on multiscale phenomena in designing engineered regulatory genetic networks and new biological technologies.

\section{References}

1. Chen, L., Wang R., Kobayashi, T. J. and Aihara K.: Dynamics of Gene Regulatory Networks with Cell Division Cycle, Phys. Rev. E, 70 (2004), 011909.

2. Jong, H.D.: Modeling and Simulation of Genetic Regulatory Systems: A Literature Review, J. of Computational Biology, 9(1) (2002), 67-103.

3. Novak, B., Pataki, Z., Ciliberto, A. and Tyson, J. J.: Mathematical Model of the Cell Division Cycle of Fission Yeast, CHAOS, 11(1) (2001), 277-286.

4. Steuer, R.: Effects of Stochasticity in Models of the Cell Cycle: from Quantized Cycle Times to Noise-induced Oscillations, J. of Theoretical Biology, 228 (2004), 293-301. 